\title{
Nonthrombotic pulmonary embolism secondary to cyanoacrylate embolization of gastric varices
}

\author{
Parthiv Amin MD, Tania Pannu MD, Rachid Mohamed MD, Kathryn Watson MD
}

Cite as: CMAJ 2022 March 7;194:E336-7. doi: 10.1503/cmaj.211203

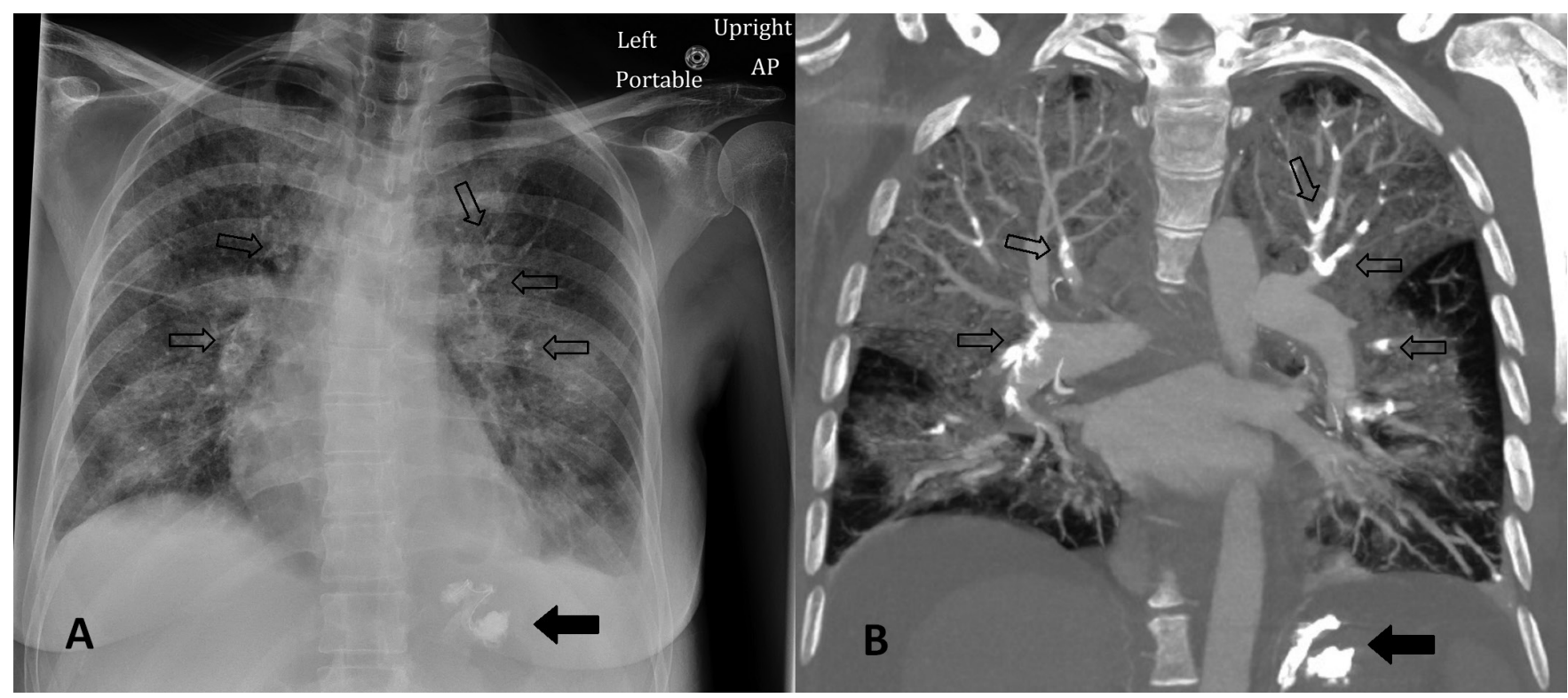

Figure 1: Chest imaging of a 51-year-old woman with bilateral nonthrombotic pulmonary embolism, performed 4 days after variceal obliteration. A) A chest radiograph shows increased interstitial markings and parenchymal opacities. B) A coronal slice from a chest computed tomography (CT) pulmonary angiogram shows multiple high-attenuation filling defects of the pulmonary arteries, compatible with nonthrombotic emboli of iodinated glue. (hollow arrows, also seen on radiograph). High-attenuation material in the left upper abdomen shows the iodinated glue and coil embolization of a gastric varix (solid arrows). Extensive ground glass attenuation on the $\mathrm{CT}$ is compatible with lipiodol-related pneumonitis.

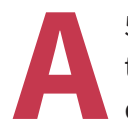

51-year-old woman with esophageal varices secondary to decompensated cirrhosis presented to the emergency department with hematemesis. Her heart rate was 123 beats/min and her blood pressure was $86 / 43 \mathrm{~mm} \mathrm{Hg}$. Her initial hemoglobin was 33 (normal $120-160$ ) g/L, her platelet count was 245 (normal 150-400) $\times 10^{9} / \mathrm{L}$ and her international normalized ratio was 1.5 (normal 0.9-1.1). We treated the patient with intravenous crystalloid, 3 units of blood, pantoprazole, octreotide and ceftriaxone. Emergent esophagogastroduodenoscopy showed new gastric varices. We performed interventional endoscopic ultrasonography with coil embolization, immediately followed by an injection of cyanoacrylate and lipiodol, and achieved variceal obliteration. One day after the procedure, the patient developed severe hypoxemia, with a respiratory rate of 20-34 breaths/min and oxygen saturation of $77 \%-81 \%$ on room air. Chest radiograph showed bilateral infiltrates. We initiated treatment with piperacillin-tazobactam for presumed aspiration pneumonitis. When she did not improve, a computed tomography pulmonary angiogram showed acute nonthrombotic pulmonary emboli and lipiodol pneumonitis (Figure 1). We treated her with intravenous steroids for 4 days. She improved rapidly and was discharged home without oxygen support.

Nonthrombotic pulmonary embolism (NTPE) is an uncommon, life-threatening complication of cyanoacrylate glue sclerotherapy, with an incidence of $0.5 \%$ to $4.3 \% .^{1}$ Endoscopic ultrasound-guided techniques with combined coil and cyanoacrylate are more efficacious than cyanoacrylate or coil alone, with low rates of NTPE $(0.7 \%)$. $^{2,3}$ Cyanoacrylate injected into gastric and esophageal varices can cause NTPE via portosystemic shunting between the varices and the gastro-spleno-renal collaterals, left renal vein, inferior vena cava, right heart and pulmonary arteries. ${ }^{4}$ 
Radiographic findings of cyanoacrylate NTPE are seen as radiopaque material outlining the pulmonary arteries. Lipiodol, an iodized oil used as a cyanoacrylate diluent, prevents premature polymerization inside the injection device. Lipiodol emboli present as consolidation, parenchymal infiltrates and high-attenuation material in the lungs. ${ }^{5}$ Lipiodol is postulated to cause pneumonitis from decomposition into free fatty acids that elicit an inflammatory injury, with capillary leakage and pulmonary edema. ${ }^{6}$

Supportive management is the preferred treatment. Despite minimal evidence, steroids can be considered to treat lipiodol pneumonitis. Anticoagulation is not indicated, as the pathophysiology of NTPE is mechanical obstruction by the glue, not thrombosis.

\section{References}

1. Saraswat VA, Verma A. Gluing gastric varices in 2012: lessons learnt over 25 years. J Clin Exp Hepatol 2012;2:55-69.

2. Bhat YM, Weilert F, Fredrick RT, et al. EUS-guided treatment of gastric fundal varices with combined injection of coils and cyanoacrylate glue: a large U.S. experience over 6 years (with video). Gastrointest Endosc 2016;83:1164-72.

3. Thiruvengadam SS, Sedarat A. The role of endoscopic ultrasound (EUS) in the management of gastric varices. Curr Gastroenterol Rep 2021;23:1.

4. Watanabe K, Kimura K, Matsutani S, et al. Portal hemodynamics in patients with gastric varices: a study in 230 patients with esophageal and/or gastric varices using portal vein catheterization. Gastroenterology 1988;95:434-40.

5. Asah D, Raju S, Ghosh S, et al. Nonthrombotic pulmonary embolism from inorganic particulate matter and foreign bodies. Chest 2018;153:1249-65.

6. Chung JW, Park JH, Im JG, et al. Pulmonary oil embolism after transcatheter oily chemoembolization of hepatocellular carcinoma. Radiology 1993; 187:689-93.

\section{Competing interests: None declared.}

This article has been peer reviewed.

The authors have obtained patient consent.

Affiliations: Departments of Radiology (Amin), Medicine (Pannu, Mohamed, Watson), Gastroenterology (Mohamed) and Emergency Medicine (Watson), University of Calgary, Calgary, Alta.

Content licence: This is an Open Access article distributed in accordance with the terms of the Creative Commons Attribution (CC BY-NC-ND 4.0) licence, which permits use, distribution and reproduction in any medium, provided that the original publication is properly cited, the use is noncommercial (i.e., research or educational use), and no modifications or adaptations are made. See: https://creativecommons.org/licenses/ by-nc-nd/4.0/

Correspondence to: Parthiv Amin, parthiv.amin@ucalgary.ca

Clinical images are chosen because they are particularly intriguing, classic or dramatic. Submissions of clear, appropriately labelled high-resolution images must be accompanied by a figure caption. A brief explanation ( 300 words maximum) of the educational importance of the images with minimal references is required. The patient's written consent for publication must be obtained before submission. 